\title{
Efecto de los factores solubles de Staphylococcus aureus, Staphylococcus capitis y Staphylococcus epidermidis sobre la funcionalidad espermática
}

\author{
Sebastián Galarzo Pardo ${ }^{1}$, Maria Alejandra Cano Cháves ${ }^{1}$, Jenniffer Puerta Suarez ${ }^{1}$, \\ Mariluz Giraldo ${ }^{2}$, B. José Manuel Mayorga ${ }^{1}$, Ángela P. Cadavid ${ }^{1}$, Walter Cardona Maya ${ }^{1}$. \\ ${ }^{1}$ Grupo Reproducción, Departamento de Microbiología y Parasitología, Facultad de Medicina, Universidad de Antioquia, \\ Medellín, Colombia. ${ }^{2}$ Escuela de Microbiología, Universidad de Antioquia, Medellín, Colombia.
}

\section{RESUMEN}

Antecedentes: La interacción entre los espermatozoides con algunas especies bacterianas o sus factores solubles influyen en el deterioro de la calidad seminal, alterando la función reproductiva del hombre. Objetivo: El objetivo de este trabajo fue determinar el efecto de los factores solubles de Staphylococcus aureus, Staphylococcus capitis y Staphylococcus epidermidis sobre la calidad seminal. Método: Los factores solubles producto del metabolismo bacteriano de las cepas de S. aureus y S. Capitis sensible a oxacilina y S. aureus y S. Epidermidis resistente a oxacilina se incubaron con las muestras de semen de 20 voluntarios y se cuantificaron los parámetros seminales convencionales y funcionales por microscopía y citometría de flujo, respectivamente. Resultados: Se observó una disminución en la movilidad espermática con los factores solubles de $\mathrm{S}$. aureus, esta disminución fue mayor con la cepa sensible y el efecto negativo sobre la movilidad fue inmediato. Al incubar los espermatozoides con los factores solubles de $\mathrm{S}$. aureus sensible a oxacilina, se afectaron todos los parámetros funcionales excepto la integridad de la cromatina y se observó menor liberación de especies reactivas de oxígeno; con los factores solubles de la cepa de S. aureus resistente a oxacilina se observó una disminución en la lipoperoxidación de membrana y en la expresión de anexina V. Conclusión: Este estudio da cuenta del efecto negativo de los factores solubles de la bacteria S. aureus tanto sensible como resistente a oxacilina sobre los parámetros espermáticos convencionales y funcionales, y por ende en su función reproductiva.

\section{PALABRAS CLAVE: Bacteria, factores solubles, calidad seminal, función espermática, Staphylococcus}

\section{SUMMARY}

Background: The interaction between sperm with some bacteria species and their soluble factors are the deterioration of semen quality by altering the reproductive function of man. Aim: The aim of this study was to determine the effect of soluble factors Staphylococcus aureus, Staphylococcus epidermidis and Staphylococcus capitis on semen quality. Methods: The soluble factors product of bacterial metabolism of the strains of $\mathrm{S}$. aureus and S. capitis methicillin sensitive and S. aureus and S. epidermidis resistant to oxacillin, were incubated with semen samples from 20 volunteers. Subsequently, conventional seminal parameters were measured and functional quantified by microscopy and flow cytometry, respectively. Results: A decrease was observed in sperm motility with soluble factors of $S$. aureus, this decrease was higher with the sensitive strain that with oxacillin resistant strain and the negative effect on motility was immediate. By incubating the sperm with soluble factor from oxacillin-sensitive $S$. aureus, all functional parameters were affected except the chromatin integrity and reduced release of reactive oxygen species, mean fluorescence intensity in 
oxacillin resistant $\mathrm{S}$. aureus strain was decrease in membrane lipid peroxidation and annexin $\mathrm{V}$ expression. Conclusions: This study reports the negative effect of soluble factors of bacteria either $\mathrm{S}$. aureus sensitive and resistant to oxacillin, over conventional and functional sperm parameters, and therefore in their reproductive function.

\section{KEY WORDS: Bacteria, soluble factors, sperm quality, sperm function, Staphylococcus}

\section{INTRODUCCIÓN}

Las infecciones del tracto urinario son la causa más común de infecciones bacterianas en el mundo y son responsables de una alta morbilidad (1). Se estima que por centro de salud, existen aproximadamente 10 casos por cada 1000 pacientes al día (2), con una prevalencia que varía de acuerdo a las especies bacterianas, siendo E. coli la más común con un $62,7 \%$, seguido del grupo de Klebsiella, Enterobacter y Serratia con $9,7 \%$ y Staphylococcus aureus con $4,0 \%$ (3). Aproximadamente la mitad de las mujeres experimentan al menos una infección del tracto urinario durante su vida (4), sin embargo, en hombres éstas son generalmente asintomáticas y se caracterizan principalmente por afectar sitios anatómicos del tracto reproductivo, como las vías urinarias, los testículos, el epidídimo y las glándulas sexuales accesorias (5), por lo que se presume que los espermatozoides durante su desarrollo, maduración o transporte pueden interactuar con algunas especies bacterianas, o los factores solubles producto del metabolismo bacteriano (6-8); inclusive se estima que al menos el $15 \%$ de los casos de infertilidad masculina son consecuencia de algún tipo de infección bacteriana (6).

Aunque no siempre la presencia de bacterias en el semen -bacteriospermia- culmina en infección, la sola existencia de algunas especies bacterianas o de los factores solubles producto de su metabolismo, pueden desencadenar una serie de efectos negativos que influyen directa o indirectamente en la disminución de la calidad seminal (9-11). Esta alteración es observada no solo en términos de la movilidad y la viabilidad, sino en los parámetros funcionales (9-19), lo cual finalmente impactaría sobre la función reproductiva masculina.

Adicionalmente, se ha demostrado que la prevalencia de bacteriospermia en hombres con alteraciones en la reproducción varía, con prevalencias que van desde el 25 al 100\% (20-22), por lo que un alto porcentaje de los casos de infertilidad idiopática, pueden deberse a la presencia de especies bacterianas difíciles de diagnosticar (8), con el agravante que estos casos de infecciones del tracto reproductivo no son de reporte obligatorio en el sistema de salud colombiano y no son evaluadas de rutina.
S. aureus es un microorganismo oportunista que coloniza la piel, las fosas nasales, las axilas, la faringe y el tracto urogenital en aproximadamente el $20 \%$ de personas sanas $(23,24)$; es la bacteria más frecuentemente aislada en las infecciones asociadas al cuidado de la salud, con el 5 a $40 \%$ de los casos (25); presenta mecanismos de resistencia a antibióticos debido a la transferencia horizontal de los genes de virulencia, que han contribuido a la aparición de cepas resistentes a oxacilina (meticilina) como las cepas portadoras del gen mecA que codifica una proteína de unión a penicilina (26). Además esta bacteria pertenece a la microbiota predominante de hombres infértiles con una disminución significativa de la movilidad espermática (27), pero son pocos los estudios que se han realizado incubando los factores solubles del metabolismo bacteriano con los espermatozoides y evaluando su efecto sobre las pruebas funcionales.

Dentro del grupo de los Staphylococcus coagulasa negativo se encuentran las especies $\mathrm{S}$. capitis y S. epidermidis, las cuales son las principales responsables de las infecciones del tracto reproductivo masculino después de $\mathrm{E}$. coli $(28,29)$. Estas bacterias poseen una serie factores de adherencia, toxinas y polipéptidos implicados en su patogenicidad tales como la hemolisina, las leucocidinas y las enterotoxinas $(25,30,31)$, que pueden influir en el detrimento de la calidad seminal.

Teniendo en cuenta que durante los procesos infecciosos las bacterias o los factores solubles producto de su metabolismo, pueden afectar la fisiología de los espermatozoides, el objetivo del estudio fue determinar el efecto de los factores solubles bacterianos de S. aureus, S. capitis y S. epidermidis sobre los parámetros seminales convencionales y funcionales.

\section{MATERIALES Y MÉTODOS}

Cepas bacterianas. Se emplearon las cepas S. aureus sensible (ATCC 29213) y resistente a la oxacilina (aislado clínico), además dos cepas de Staphylococcus coagulasa negativo (aislados clínicos): S. capitis sensible y S. epidermidis resistente a oxacilina. La cepa de S. epidermidis fue resistente al tratamiento con ciprofloxacino, levofloxacino, eritromicina, clindamicina y trimetoprima/sulfametoxazol. 
Obtención de los factores solubles. Los factores solubles de las cuatro cepas de bacterias, se obtuvieron incubando durante 30 minutos un inóculo de cada bacteria a una concentración de 0,5 Macfarland en solución salina. Posteriormente, el medio se centrifugó a $600 \mathrm{~g} / 10$ minutos, se recolectó el sobrenadante y se filtró por una membrana de 0,2 um (Advantec Industries, China), se alicuotó y se almacenó a $-20^{\circ} \mathrm{C}$ hasta su uso.

Muestras de semen. Se emplearon 20 muestras de semen de voluntarios aparentemente sanos, mayores de 18 años con parámetros seminales iguales o superiores a los límites inferiores de referencia establecidos por la Organización Mundial de la Salud (OMS) $(32,33)$. Las muestras de semen fueron colectadas por masturbación en un recipiente estéril, después de una abstinencia sexual de 3 a 5 días y analizadas máximo 2 horas después de su recolección. A cada muestra de semen se le determinaron los parámetros seminales convencionales como el volumen, la movilidad, la viabilidad (33) y la concentración usando la cámara de Makler (Sefi-Medical Instruments, Israel) (34).

Evaluación de los factores solubles de las bacterias sobre la movilidad espermática y sobre los parámetros funcionales. Se realizó la incubación de los espermatozoides con los factores solubles en proporción 1 a 1,50 $\mu \mathrm{L}$ de semen y 50 $\mu \mathrm{L}$ de factores solubles en un volumen final de 100 $\mu \mathrm{L}$, a $37^{\circ} \mathrm{C} / 60$ minutos con el fin de determinar por microscopía su efecto sobre la movilidad espermática, evaluada cada 15 minutos durante un lapso de una hora. Los resultados son expresados como porcentaje de cambio en la movilidad, que es la relación entre el porcentaje de movilidad con los factores solubles respecto al control. Adicionalmente, se incubaron $150 \mu \mathrm{L}$ de semen con $150 \mu \mathrm{l}$ de los diferentes factores a $37^{\circ} \mathrm{C} / 1$ hora, se tomaron alícuotas de $2 \times 10^{6}$ espermatozoides para evaluar cada uno de los siguientes parámetros funcionales: potencial de membrana mitocondrial, integridad de la membrana plasmática, lipoperoxidación de la membrana, fragmentación de la cromatina, detección temprana de apoptosis y producción intracelular de especies reactivas de oxígeno (ERO) mediante citometría de flujo (CoulterEpics XL BeckmanCoulter, CA, USA) analizando entre 5.000 y 10.000 espermatozoides.

Detección del potencial de membrana mitocondrial espermática. Los espermatozoides se incubaron con ioduro de propidio (IP, Molecular Probes®Inc, OR, USA) [0.25mg/mL] y 3,3'dihexi-

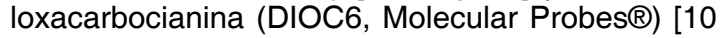
$\mathrm{nM}$ ] a $37^{\circ} \mathrm{C} / 30$ minutos; los espermatozoides se lavaron una vez centrifugando a $300 \mathrm{~g} / 5$ minutos. El botón fue resuspendido en PBS y se realizó la lectura en el citómetro de flujo como había sido previamente estandarizado en el Grupo Reproducción (19).
Determinación de la integridad de la membrana espermática. Siguiendo el protocolo previamente establecido (19), los espermatozoides se incubaron mezclaron con IP (concentración final $0,25 \mathrm{mg} / \mathrm{mL}$ ) y Sybr $14[1 \mu \mathrm{M}]$ (LIVE/DEAD® SpermViability Kit, Molecular Probes $\left(\right.$ )a $37^{\circ} \mathrm{C} / 30$ minutos, se lavaron una vez con PBS, se resuspendió el botón y se realizó la lectura en el citómetro de flujo.

Análisis de la lipoperoxidación de la membrana espermática. Se incubaron los espermatozoides con 4,4-difluoro-4-bora-3a-4a-diaza-s-indaceno (BODIPY C11Molecular Probes®Inc, OR, USA) a $37^{\circ} \mathrm{C} / 30$ minutos, los espermatozoides se lavaron una vez y fueron resuspendidos en PBS antes de realizar la lectura en el citómetro de flujo (14).

Evaluación de los niveles de especies reactivas de oxígeno intracelulares espermáticas. Los espermatozoides se incubaron con di-acetato de 2',7' diclorofluoresceína (DCFH-DA, Sigma-Aldrich, St Louis, MO, USA) [1 $\mu \mathrm{M}]$ y IP $[0,25 \mathrm{mg} / \mathrm{mL}]$ a $37^{\circ} \mathrm{C} / 5$ minutos, se lavaron 3 veces, el botón fue resuspendido en PBS y se realizó la lectura en el citómetro de flujo siguiendo el protocolo previamente establecido (19).

Detección de la integridad de la cromatina espermática. Se diluyeron los espermatozoides en buffer TNE (TRIS-HCl, NaCl, EDTA - disódica, $\mathrm{pH}$ : $7,4)$. Justo antes de leer la muestra en el citómetro de flujo, se adicionó $400 \mu \mathrm{L}$ de solución detergente ácida $(\mathrm{HCl}, \mathrm{NaCl}$, Tritón X-100, pH: 1,2) y 30 segundos después se adicionó la solución colorante naranja de acridina (Sigma-Aldrich, St Louis, MO, USA, $0.006 \mathrm{mg} / \mathrm{mL}$ ), finalmente se realizó la lectura en el citómetro de flujo siguiendo el protocolo previamente establecido (17-19,35).

Detección de eventos apoptóticos tempranos. A la suspensión de espermatozoides se les agregó el buffer de unión a una concentración de 1X (10X Annexin V Binding Buffer, Sigma-Aldrich Co, St Louis, MO, USA), se centrifugó a $300 \mathrm{~g} / 5 \mathrm{mi}-$ nutos y se descartó el sobrenadante. Se adicionaron $3 \mu \mathrm{L}$ de Anexina V FITC, se incubó a $37^{\circ} \mathrm{C} / 30$ minutos y se agregó $1,5 \mu \mathrm{L}$ de IP incubando durante 5 minutos, se lavó una vez con con PBS (Gib$C O \circledR, N Y, U S A)$ y se realizó la lectura en el citómetro de flujo (13).

Análisis de datos. Los resultados de las citometrías de flujo de cada parámetro funcional fueron graficados y procesados utilizando los programas WinMDI 2.9 (The Scripps Research Institute, La Jolla - CA, USA) y Flow Jo 7.6 (Tree Star, Inc. Oregon, USA).En este estudio se aplicó estadística descriptiva (mediana y RIC -rango intercuartil-). Los resultados obtenidos se analizaron con estadística no paramétrica utilizando la prueba Wilcoxon, considerando significancia estadística un $p<0,05$, utilizando el programa Graph Pad Prism 5 (Graphpad, CA, USA). 


\section{RESULTADOS}

Al incubar los espermatozoides humanos con los factores solubles producto del metabolismo de la bacteria $\mathrm{S}$. aureus, se observó que los factores de la cepa sensible a oxacilina disminuyeron la movilidad desde un $25 \%$ a los 15 minutos, hasta aproximadamente un $40 \%$ a los 60 minutos respecto al control. Con los factores de la cepa resistente a oxacilina, el efecto negativo sobre la movilidad fue inmediato, observando una disminución del $20 \%$ que se mantuvo estable en función del tiempo desde los 15 segundos hasta una hora de incubación (Figura 1a).

Los factores solubles de la bacteria S. capitis sensible a oxacilina disminuyeron en un $30 \%$ la movilidad a los 30 minutos y el efecto se mantuvo en el tiempo. Por su parte, los espermatozoides incubados con los factores solubles de la cepa $S$. epidermidis resistente a oxacilina, presentaron un mayor deterioro en la movilidad a los 15 minutos de incubación y este efecto se mantuvo en un rango de $30 \%$ (Figura $1 \mathrm{~b}$ ).

El efecto de los factores solubles producto del metabolismo de las bacterias tanto sensibles como resistentes a oxacilina, sobre los parámetros funcionales de los espermatozoides se consolida en la Tabla I. Aunque las diferencias entre estas poblaciones con respecto al control, no fueron estadísticamente significativas se observaron cambios en el efecto de los factores solubles de cada cepa bacteriana entre ambas especies, siendo mayores los efectos de los factores solubles de la cepa S.

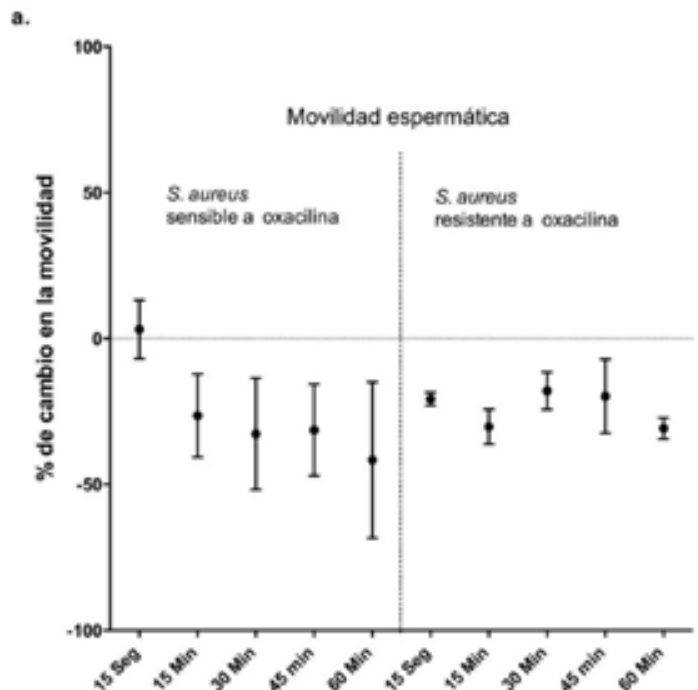

aureus tanto sensible como resistente a oxacilina.

Con respecto a la evaluación del potencial de membrana mitocondrial con DIOC6, los factores solubles de $\mathrm{S}$. aureus sensible a oxacilina indujeron una disminución del potencial de membrana mitocondrial alto con respecto al control. Por su parte la determinación de la integridad de la membrana con el colorante SYBR evidenció un efecto negativo de los factores solubles de $\mathrm{S}$. aureus sensible a oxacilina sobre las células viables, moribundas y necróticas respecto a su control (Tabla I).

Al evaluar la producción de especies reactivas de oxígeno en los espermatozoides incubados con los factores solubles de las cepas sensibles y resistentes de S. aureus y Staphylococcus coagulasa negativo, no se encontraron diferencias, sin embargo las intensidades medias de fluorescencia (IMF) disminuyeron con respecto al control.

Adicionalmente, se observó diferencias en el efecto de los factores solubles de $\mathrm{S}$. aureus sobre la lipoperoxidación de la membrana celular de los espermatozoides, la cepa resistente presentó un mayor efecto que la sensible, y de ambas frente al control, aunque no fueron estadísticamente significativas. En la evaluación de la integridad de la cromatina espermática, no se observaron diferencias en el efecto de los factores solubles de las bacterias con respecto a su control. Finalmente, al utilizar anexina $\mathrm{V}$ como indicador de un estado proapoptótico, se encontraron diferencias en los tratamientos con los factores solubles de $\mathrm{S}$. aureus, tanto en las cepas sensible como la resistente, y de ambas frente al control.

Figura 1 a $y$ b. Efecto de los factores solubles producto del metabolismo de las bacterias sobre la movilidad espermática.

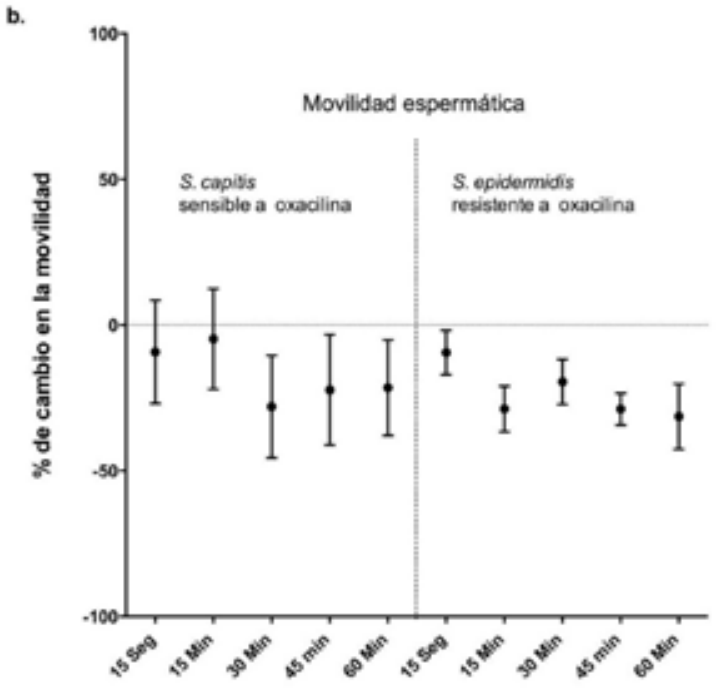




\section{Tabla I}

\section{EFECTO DE LOS FACTORES SOLUBLES PRODUCTO DEL METABOLISMO DE LAS BACTERIAS SOBRE LOS PARÁMETROS ESPERMÁTICOS FUNCIONALES}

\begin{tabular}{|c|c|c|c|c|c|c|}
\hline \multirow[b]{2}{*}{ Parámetro } & \multicolumn{3}{|c|}{$\begin{array}{l}\text { Staphylococcus aureus } \\
\text { Mediana (rango) }\end{array}$} & \multicolumn{3}{|c|}{$\begin{array}{l}\text { Staphylococcus coagulasa negativo } \\
\text { Mediana (rango) }\end{array}$} \\
\hline & Control & $\begin{array}{l}\text { Sensible a } \\
\text { oxacilina }\end{array}$ & $\begin{array}{c}\text { Resistente a } \\
\text { oxacilina }\end{array}$ & Control & $\begin{array}{l}\text { S. capitis } \\
\text { sensible a } \\
\text { oxacilina }\end{array}$ & $\begin{array}{l}\text { S. epidermidis } \\
\text { resistente a } \\
\text { oxacilina }\end{array}$ \\
\hline Células con PMM Alto (\%) & $\begin{array}{c}51,7 \\
(42,6-71,6)\end{array}$ & $\begin{array}{c}42,5 \\
(25,0-72,9)\end{array}$ & $\begin{array}{c}48,1 \\
(44,8-72,4)\end{array}$ & $\begin{array}{c}50,1 \\
(41,5-57,1)\end{array}$ & $\begin{array}{c}53,5 \\
(35,0-54,3)\end{array}$ & $\begin{array}{c}46,2 \\
(37,8-58,3)\end{array}$ \\
\hline Células con PMM Bajo (\%) & $\begin{array}{c}7,7 \\
(4,8-33,5)\end{array}$ & $\begin{array}{c}14,0 \\
(4,2-42,4)\end{array}$ & $\begin{array}{c}8,4 \\
(3,2-27,4)\end{array}$ & $\begin{array}{c}15,2 \\
(13,6-20,1)\end{array}$ & $\begin{array}{c}14,8 \\
(13,7-15,8)\end{array}$ & $\begin{array}{c}12,8 \\
(11,8-21,8)\end{array}$ \\
\hline Células necróticas (\%) & $\begin{array}{c}23,9 \\
(18,4-42,4)\end{array}$ & $\begin{array}{c}32,6 \\
(16,7-42,2)\end{array}$ & $\begin{array}{c}24,3 \\
(14,7-46,6)\end{array}$ & $\begin{array}{c}29,4 \\
(24,7-44,8)\end{array}$ & $\begin{array}{c}30,5 \\
(26,7-51,2)\end{array}$ & $\begin{array}{c}30,3 \\
(28,0-49,2)\end{array}$ \\
\hline $\begin{array}{l}\text { Células con membrana } \\
\text { celular íntegra (\%) }\end{array}$ & $\begin{array}{c}73,7 \\
(48,4-73,9)\end{array}$ & $\begin{array}{c}66,3 \\
(45,6-69,9)\end{array}$ & $\begin{array}{c}69,3 \\
(39,1-73,3)\end{array}$ & $\begin{array}{c}65,2 \\
(43,3-67,6)\end{array}$ & $\begin{array}{c}63,7 \\
(62,5-65,1)\end{array}$ & $\begin{array}{c}65,5 \\
(61,7-70,0)\end{array}$ \\
\hline Células moribundas (\%) & $\begin{array}{c}3,7 \\
(3,4-4,0)\end{array}$ & $\begin{array}{c}6,8 \\
(3,0-20,5)\end{array}$ & $\begin{array}{c}3,1 \\
(1,1-28,6)\end{array}$ & $\begin{array}{c}7,0 \\
(6,9-11,3)\end{array}$ & $\begin{array}{c}8,9 \\
(6,2-9,3)\end{array}$ & $\begin{array}{c}7,1 \\
(2,5-13,0)\end{array}$ \\
\hline Células necróticas (\%) & $\begin{array}{c}20,7 \\
(19,5-37,5)\end{array}$ & $\begin{array}{c}24,7 \\
(24,6-32,8)\end{array}$ & $\begin{array}{c}23,7 \\
(22,7-30,5)\end{array}$ & $\begin{array}{c}25,6 \\
(23,6-43,6)\end{array}$ & $\begin{array}{c}26,7 \\
(24,6-28,1)\end{array}$ & $\begin{array}{c}24,8 \\
(24,1-25,4)\end{array}$ \\
\hline $\begin{array}{l}\text { Células positivas para } \\
\text { DCFH DA (\%) }\end{array}$ & $\begin{array}{c}71,5 \\
(62,4-84,0)\end{array}$ & $\begin{array}{c}69,3 \\
(53,2-80,7)\end{array}$ & $\begin{array}{c}72,7 \\
(57,1-78,3)\end{array}$ & $\begin{array}{c}68,3 \\
(51,7-70,8)\end{array}$ & $\begin{array}{c}65,3 \\
(50,0-69,3)\end{array}$ & $\begin{array}{c}65,9 \\
(55,6-69,2)\end{array}$ \\
\hline $\begin{array}{l}\text { IMF de células positiva } \\
\text { para DCFH DA }\end{array}$ & $\begin{array}{c}8,2 \\
(3,3-10,7)\end{array}$ & $\begin{array}{c}5,4 \\
(2,8-7,1)\end{array}$ & $\begin{array}{c}5,1 \\
(2,3-6,3)\end{array}$ & $\begin{array}{c}2,1 \\
(1,7-2,8)\end{array}$ & $\begin{array}{c}2,1 \\
(1,7-2,6)\end{array}$ & $\begin{array}{c}2,4 \\
(2,3-2,5)\end{array}$ \\
\hline $\begin{array}{l}\text { Células positivas para } \\
\text { lipoperoxidación de } \\
\text { membrana (\%) }\end{array}$ & $\begin{array}{c}4,7 \\
(0,0-4,9)\end{array}$ & $\begin{array}{c}3,1 \\
(0,1-4,6)\end{array}$ & $\begin{array}{c}1,9 \\
(0,1-2,8)\end{array}$ & $\begin{array}{c}3,5 \\
(2,9-3,5)\end{array}$ & $\begin{array}{c}3,7 \\
(2,6-5,4)\end{array}$ & $\begin{array}{c}3,9 \\
(0,9-4,2)\end{array}$ \\
\hline $\begin{array}{l}\text { Células positivas para } \\
\text { Anexina } \vee(\%)\end{array}$ & $\begin{array}{c}1,2 \\
(0,7-1,8)\end{array}$ & $\begin{array}{c}3,3 \\
(0,9-5,7)\end{array}$ & $\begin{array}{c}3,6 \\
(2,5-4,8)\end{array}$ & $\begin{array}{c}0,2 \\
(0,1-0,5)\end{array}$ & $\begin{array}{c}0,2 \\
(0,2-0,5)\end{array}$ & $\begin{array}{c}0,3 \\
(0,2-0,6)\end{array}$ \\
\hline $\begin{array}{l}\text { Índice de fragmentación } \\
\text { de ADN }\end{array}$ & $\begin{array}{c}4,97 \\
(3,3-6,1)\end{array}$ & $\begin{array}{c}4,68 \\
(4,4-6,8)\end{array}$ & $\begin{array}{c}4,85 \\
(4,3-6,8)\end{array}$ & $\begin{array}{c}8,03 \\
(7,5-8,3)\end{array}$ & $\begin{array}{c}7,46 \\
(7,4-9,1)\end{array}$ & $\begin{array}{c}7,40 \\
(6,6-7,7)\end{array}$ \\
\hline
\end{tabular}

PMM: Potencial de membrana mitocondrial. IMF: Intensidad media de fluorescencia.

\section{DISCUSIÓN}

En este estudio se evaluó el efecto de los factores solubles de las bacterias $\mathrm{S}$. aureus, sensible y resistente a oxacilina y dos cepas de Staphylococcus coagulasa negativo, S. capitis sensible y S. epidermidis resistente a oxacilina, sobre la calidad espermática de muestras seminales de individuos aparentemente sanos.

La resistencia a oxacilina (meticilina) se ha convertido en un importante problema de salud pública y su prevalencia está aumentando a nivel mundial. Por ejemplo España pasó de 1,5\% en 1986 a 29,2\% en 2006 para S. aureus, mientras que Staphylococcus coagulasa negativo tuvo un cambio en la prevalencia de $32,5 \%$ en 1986 a $66,7 \%$ en $2006(36,37)$. Por esta razón los controles en las entidades de salud son cada vez más rigurosos, por la complejidad que genera el tratamiento de las bacterias del género Staphylococcus que adquieren por transmisión horizontal el gen mecA que lo hacen resistente a una gran variedad de los antibióticos disponibles actualmente $(26,38)$. 
El grupo de los Staphylococcus que adquieren el gen mecA resistente a la oxacilina se han convertido en un principal foco de atención debido a su presencia en el tracto urogenital y el carácter asintomático de las infecciones por estos microorganismos en hombres $(26,38)$. Los microorganismos que están presentes en el tracto urogenital masculino pueden estar afectando negativamente la calidad del espermatozoide $(7,8)$ como ha sido reportado en individuos positivos para la infección por el VIH (39-41), sin embargo no se conocen los mecanismos de acción de las toxinas y factores de adherencia de las bacterias del género Staphylococcus sobre la calidad espermática. No obstante, otro microorganismo patógeno y con alta incidencia en el tracto genital masculino es E. coli, el cual ha sido estudiado por su capacidad aglutinante a través de sus factores solubles, como son las fimbrias $P$ y que inhiben la movilidad espermática hasta un $45 \%$ mediado por los receptores de manosa (42); estos receptores son las principales proteínas de unión entre los espermatozoides y las estructuras bacterianas que generan una pérdida de la movilidad de las células espermáticas (43). La concentración bacteriana produce efectos negativos en los parámetros convencionales espermáticos debido a que a mayor concentración bacteriana es mayor el detrimento de los parámetros (5). Estudios previos han demostrado una disminución de la calidad espermática y la infertilidad masculina cuando se da el contacto entre los factores solubles de E. coli y los espermatozoides $(9,11)$.

En el presente estudio, la movilidad de los espermatozoides incubados con los factores solubles de la cepa S. aureus, mostraron una tendencia a la disminución y ésta fue mayor con la cepa sensible a oxacilina. S. aureus causó un detrimento en la movilidad espermática, no obstante, el hecho de que esta bacteria resistente cause un efecto negativo inmediato hace pensar que durante su crecimiento libera una alta concentración de moléculas al medio como exopolisacáridos, residuos de la cápsula, adhesinas (30), alfa-toxinas, leucocidinas y enterotoxinas (44) que puedan estar afectando la calidad de los espermatozoides.

Con respecto a $\mathrm{S}$. capitis y $\mathrm{S}$. epidermidis se observó variabilidad en los datos lo cual podría deberse a que el efecto causado por estas bacterias no sea permanente y que el espermatozoide podría estar generando una estrategia para desprenderse de los factores solubles que en muchos casos pueden estar causando aglutinación o daños en el potencial de membrana, en la lipoperoxidación y en la liberación de ERO. Resultados similares se muestra en estudios previos con otros microorganismos bacterianos en los que el lipopolisacárido que puede estar presente en los factores solubles, induce la liberación de especies reactivas de oxígeno y por ende en la pérdida de movilidad de los espermatozoides (45); además factores solubles de E. coli causaron disminución del potencial de membrana mitocondrial y translocación de la fosfatidilserina (11).

Continuando con los parámetros funcionales, se evidencia un efecto inhibidor sobre la movilidad espermática ejercida por los factores solubles de $\mathrm{S}$. aureus sensible a oxacilina relacionada con el potencial de membrana mitocondrial y con la integridad de membrana, pero no se relaciona con la liberación de ERO, ni con la liperoxidación lipídica aunque estos se han relacionado en otros estudios con bacterias como E. coli (9). Los factores solubles de $\mathrm{S}$. aureus sensible a oxacilina causaron disminución en el potencial de membrana mitocondrial, en la integridad de membrana y aumento del número de células necróticas. Aunque los resultados no sean estadísticamente significativos se pueden observar tendencias hacia el daño que producen los factores solubles de las cepas evaluadas sobre la movilidad espermática. Con respecto a los resultados de las cepas del grupo de los Staphylococcus coagulasa negativo no se observó una disminución de los parámetros funcionales espermáticos al incubarlos con los factores solubles.

\section{CONCLUSIÓN}

Aunque no hubo diferencias estadísticamente significativas en el efecto sobre los parámetros de los espermatozoides incubados con los factores solubles de las bacterias $S$. aureus, $S$. capitis y S. epidermidis, los resultados del estudio sugieren que la interacción de los espermatozoides humanos con los factores solubles de estas bacterias pueden afectar la calidad espermática y por ende la función reproductiva masculina.

AGRADECIMIENTOS: Este trabajo fue financiado por Colciencias (111556933373) y por la Estrategia de Sostenibilidad 2014-2015, Grupo Reproducción, de la Universidad de Antioquia. Jenniffer Puerta Suárez fue Joven Investigadora de Colciencias.

\section{REFERENCIAS}

1. Córdova E, Lespada MI, Cecchini D, Jacob N, Gomez N, Gutfraind G, et al. Prevalencia de gérmenes multirresistentes en infecciones del tracto urinario de la comunidad y asociadas a los cuidados de la salud. Actualizaciones en SIDA e Infectología. 2014;22(84):33-8.

2. Johansen TEB, Cek M, Naber K, Stratchounski L, Svendsen MV, Tenke P, et al. Prevalence of hospital-acquired urinary tract infections in urology departments. Eur Urol. 2007;51(4):1100-12. 
3. Chiavassa L, Vaschalde G. Prevalencia y perfil de resistencia de microorganismos en infecciones del tracto urinario. Bioquim Patol Clin. 2008;72(3):11-8.

4. Dhakal B, Kulesus R, Mulvey M. Mechanisms and consequences of bladder cell invasion by uropathogenic Escherichia coli. Eur J Clin Invest. 2008;38(s2):2-11.

5. Diemer T, Weidner W, Michelmann H, Schiefer $H$, Rovan E, Mayer F. Influence of Escherichia coli on motility parameters of human spermatozoa in vitro. Int J Androl. 1996;19(5):271-7.

6. Pellati D, Mylonakis I, Bertoloni G, Fiore C, Andrisani A, Ambrosini $G$, et al. Genital tract infections and infertility. Eur J Obstet Gynecol Reprod Biol. 2008;140(1):3-11.

7. Puerta-Suárez J, Giraldo M, Cadavid A, Cardona-Maya $\mathrm{W}$. Infecciones bacterianas del tracto reproductivo masculino y su papel en la fertilidad. Rev Chil Obstet Ginecol. 2014;79(3):209-17

8. Puerta-Suárez J, Villegas-Castaño A, Serna-Quintana G, Martínez A, Romero-Palacio J, Giraldo M, et al. Espermocultivo: crecimiento bacteriano del eyaculado y su relación con los parámetros seminales. Rev Chil Obstet Ginecol. 2015;80(1):33-40

9. Barbonetti A, Vassallo MRC, Cinque B, Filipponi S, Mastromarino P, Cifone MG, et al. Soluble products of Escherichia coli induce mitochondrial dysfunction-related sperm membrane lipid peroxidation which is prevented by lactobacilli. PloS One. 2013;8(12):e83136.

10. Fraczek M, Szumala-Kakol A, Jedrzejczak P, Kamieniczna M, Kurpisz M. Bacteria trigger oxygen radical release and sperm lipid peroxidation in in vitro model of semen inflammation. Fertil Steril. 2007;88(4):107685.

11. Schulz M, Sánchez R, Soto L, Risopatrón J, Villegas $\mathrm{J}$. Effect of Escherichia coli and its soluble factors on mitochondrial membrane potential, phosphatidylserine translocation, viability, and motility of human spermatozoa. Fertil Steril. 2010;94(2):619-23.

12. Sharma RK, Agarwal A. Role of reactive oxygen species in male infertility. Urol. 1996;48(6):835-50.

13. Glander H-J, Schaller J. Binding of annexin V to plasma membranes of human spermatozoa: a rapid assay for detection of membrane changes after cryostorage. Mol Hum Reprod. 1999;5(2):109-15.

14. Aitken R. Sperm function tests and fertility. Int J Androl. 2006;29(1):69-75.

15. Cardona-Maya W, Bergudo J, De los Rios JA, Cadavid $A$. Functional evaluation of sperm in Colombian fertile men. Arch Esp Urol. 2007;60(7):827.

16. Aitken RJ, Wingate JK, De luliis GN, McLaughlin EA. Analysis of lipid peroxidation in human spermatozoa using BODIPY C11. Mol Hum Reprod. 2007;13(4):203-11.

17. Gil-Villa AM, Cardona-Maya W, Agarwal A, Sharma $\mathrm{R}$, Cadavid A. Role of male factor in early recurrent embryo loss: do antioxidants have any effect? Fertil Steril. 2009;92(2):565-71.

18. Gil-Villa AM, Cardona-Maya W, Agarwal A, Sharma R, Cadavid Á. Assessment of sperm factors possibly involved in early recurrent pregnancy loss. Fertil Steril. 2010;94(4):1465-72.

19. Mayorga-Torres B, Cardona-Maya W, Cadavid A, Camargo M. Evaluation of sperm functional parameters in normozoospermic infertile individuals. Actas Urol Esp. 2013;37(4):221-7.
20. Henkel R, Schill WB. Sperm separation in patients with urogenital infections. Andrologia. 1998;30(S1):91-7.

21. Liversedge N, Jenkins J, Keay S, McLaughlin E, AlSufyan $\mathrm{H}$, Maile L, et al. Antibiotic treatment based on seminal cultures from asymptomatic male partners in in-vitro fertilization is unnecessary and may be detrimental. Hum Reprod. 1996;11(6):1227-31.

22. Onemu S, Ibeh I. Studies on the significance of positive bacterial semen cultures in male fertility in Nigeria. Int J Fertil Womens Med. 2000;46(4):210-4.

23. Gupta S, Prabha V. Human sperm interaction with Staphylococcus aureus: a molecular approach. J Pathog. 2012;2012.

24. Sandel MK, McKillip JL. Virulence and recovery of Staphylococcus aureus relevant to the food industry using improvements on traditional approaches. Food Control. 2004;15(1):5-10.

25. Baba-Moussa L, Anani L, Scheftel J, Couturier M, Riegel P, Haikou N, et al. Virulence factors produced by strains of Staphylococcus aureus isolated from urinary tract infections. J Hosp Infect. 2008;68(1):32-8.

26. Enright MC, Robinson DA, Randle G, Feil EJ, Grundmann H, Spratt BG. The evolutionary history of methicillin-resistant Staphylococcus aureus (MRSA). PNAS. 2002;99(11):7687-92.

27. Jiang J, Lu D. Detection of bacteria from semen of infertile males and their seminal parameters. Chin $\mathrm{J}$ Androl. 1996;10:196-8.

28. Nabi A, Khalili MA, Halvaei I, Ghasemzadeh J, Zare E. Seminal bacterial contaminations: Probable factor in unexplained recurrent pregnancy loss. Iran J Reprod Med. 2013;11(11):925.

29. Sanocka-Maciejewska D, Ciupińska M, Kurpisz M. Bacterial infection and semen quality. J Reprod Immunol. 2005;67(1):51-6.

30. Rupp ME, Archer GL. Coagulase-negative staphylococci: pathogens associated with medical progress. Clin Infect Dis. 1994:231-43.

31. Novick RP, Muir TW. Virulence gene regulation by peptides in staphylococci and other Gram-positive bacteria. Curr Opin Microbiol. 1999;2(1):40-5.

32. Cardona-Maya W. World Health Organization manual for the processing of human semen-2010. Actas Urol Esp. 2010;34(7):577-8.

33. WHO. WHO laboratory manual for the examination and processing of human semen. 2010.

34. Cardona-Maya W, Berdugo J, Cadavid A. Comparación de la concentración espermática usando la cámara de Makler y la cámara de Neubauer. Actas Urol Esp. 2008;32(4):443-5.

35. Rodríguez E, Gil-Villa AM, Aguirre-Acevedo DC, Cardona-Maya W. Evaluation of atypical semen parameters in individuals whose couples had a history of early recurrent embryo death: in search for a reference value. Biomedica. 2011;31(1):100-7.

36. Cercenado E, Sánchez-Carrillo C, Alcalá L, Bouza E. Situación actual de la resistencia de Staphylococcus en España. Cuarto estudio nacional (1996). Rev Clin Esp. 1997;197:12-8.

37. Cuevas Ó, Cercenado E, Goyanes MJ, Vindel A, Trincado $\mathrm{P}$, Boquete $\mathrm{T}$, et al. Staphylococcus spp. en España: situación actual y evolución de la resistencia a antimicrobianos (1986-2006). Enferm Infecc Microbiol Clin. 2008;26(5):269-77. 
38. Londoño JF, Ortiz GM, Gaviria A. Prevalence of personnel of the intensive of Medellín 2004. Infection. 2006;10(3):160-6.

39. Cardona-Maya W, López-Herrera A, Velilla-Hernández P, Rugeles MT, Cadavid ÁP. The Role of Mannose Receptor on HIV1 Entry into Human Spermatozoa. Am J Reprod Immunol. 2006;55(4):241-5.

40. Cardona-Maya W, Velilla P, Montoya CJ, Cadavid A, Rugeles MT. Presence of HIV-1 DNA in spermatozoa from HIV-positive patients: changes in the semen parameters. Curr HIV Res. 2009;7(4):418-24.

41. Cardona-Maya W, Velilla PA, Montoya CJ, Cadavid A, Rugeles MT. In vitro human immunodeficiency virus and sperm cell interaction mediated by the mannose receptor. J Reprod Immunol. 2011;92(1):1-7.
42. Monga M, Roberts JA. Spermagglutination by Bacteria: Receptor-specific Interactions. J Androl. 1994;15(2):151-6.

43. Wolff H, Panhans A, Stolz W, Meurer M. Adherence of Escherichia coli to sperm: a mannose mediated phenomenon leading to agglutination of sperm and $\mathrm{E}$. coli. Fertil Steril. 1993;60(1):154-8.

44. Otto M. Staphylococcus aureus toxins. Curr Opin Microbiol.. 2014;17:32-7.

45. Urata K, Narahara H, Tanaka Y, Egashira T, Takayama F, Miyakawa I. Effect of endotoxin-induced reactive oxygen species on sperm motility. Fertil Steril. 2001;76(1):163-6. 